\title{
25
}

\section{Spatial Point Pattern Analysis for Water Mains Failures in Sanandaj, Iran}

\author{
Ahmad Asnaashari, Isam Shahrour, M. Haji Sotudeh and \\ Raed Jafar
}

This chapter addresses a methodology for using spatial and statistical analysis to discover the distribution of water mains failures in space. It provides a number of new ways of looking at water mains failure data and geographical relations among them. Points are used to indicate spatial occurrences of failures and their pattern. Data from Sanandaj city in Iran over a ten year period is analysed, including 395 mains failure. Point pattern analysis is applied to exploring spatial variation and identifying whether occurrences are interrelated. Measures of central tendency scores like mean center, weighted mean center and dispersion scores such as standard deviation distance and standard deviation ellipse help explain the level of dispersion in failure data. In addition, nearest neighbor analysis and quadrat analysis were performed to find the spatial pattern in the point distribution. The findings confirm that water mains failures tend to occur in cluster. This will enable more effective management of the water distribution network by ensuring that mains are rehabilitated or replaced in cluster areas.

\subsection{Introduction}

Failure in a water distribution system constitutes a major issue for both the water authority and society. The effects are not only a disrupted service but also a significant loss of water. In countries where water is scarce, such as

Asnaashari, A., I. Shahrour, M. Haji Sotudeh and R. Jafar. 2007. "Spatial Point Pattern Analysis for Water Mains Failures in Sanandaj, Iran." Journal of Water Management Modeling R227-25. doi:

10.14796/JWMM.R227-25.

(C) CHI 2007 www.chijournal.org ISSN: 2292-6062 (Formerly in Contemporary Modeling of Urban Water Systems. ISBN: 0-9736716-3-7) 
Iran, loss of water as a result of mains failure is of particular concern. Knowledge of failure behaviour in spatial terms allows the utilities to predict the occurrence of future failures.

Traditionally, water utilities used methods such as placing pins representing failure events onto a wall map. This method was not very useful in analysing and monitoring failures. With the appearance of Geographic Information System (GIS) applications, the point mapping method is largely used for the construction of geographic maps of failure. Since the locations of failure are the variables of interest and consist of a finite number of locations observed in a spatial region, point pattern analysis has significant potential in failure surveillance. This allows engineers to understand where failure is occurring, where the failures are concentrated, and if there is any spatial pattern. To know whether observed spatial pattern is the result of chance or a systematic process, we will measure spatial distributions.

In practice, identification and analysis of a geographic concentration in pipeline failure can play an important role in improving the efficiency of preventative actions. For example, many researchers have shown that the probability of a water-main break is highest in the vicinity of a previous break (Goulter, 1993; O'Day, 1989). The ability to quantitatively determine the area of concentrated pipeline failures allows more careful evaluation of where the next failure is most likely to occur, in which direction the failure patterns may have been shifted, and why failure occurs in specific zones.

This chapter constitutes the application of GIS and appropriate spatial analysis procedures to the study of aggregate water mains failure pattern in Sanandaj city, Iran. It also outlines how this tool can be used to prioritize future rehabilitation and replacement projects.

\subsection{Background}

The analysis of point event patterns in geography, health, biology, crime, vehicle accidents, ecology and epidemiology has a long tradition (e.g. Clark \& Evans, 1954; Harvey, 1966; Mantel, 1967; Cliff \& Ord, 1981; Levine et al., 1995; Rogerson \& Sun, 2001). Point pattern analysis is concerned with the location of events, and with answering questions about the distribution of those locations, specifically whether they are clustered, randomly or regularly distributed (Cressie, 1993). The objective of a point pattern analysis may involve test of complete spatial randomness, estimation of intensity and stochastic model fitting to provide an explanation of the 
underlying processes. Applications of the approach have been successfully used in analyses of water utilities, but more studies have been performed in urban water quality issues than for pipe failures. Y. Hu and D.W. Hubble (2005) carried out simple spatial analysis and visualization of asbestos cement water main breaks pattern in the city of Regina, Canada, and observed an uneven spatial and temporal pattern. Pipe breakage was concentrated in a few subdivisions such as the central and central-west areas. In 2004, Lienyao et al. applied cluster analysis to determine the spatial diversity of chlorine residuals in the distribution system of Feng-Yuan city which was correlated with the daily lifestyle of the inhabitants and commercial activities. From the results of cluster analysis, the study area was classified into three groups: high-chlorine-level zone, medium-chlorinelevel zone, and low-chlorine-level zone. The work of Saraf et al. (2003) supports various spatial statistical operations like central tendency and dispersion on the epicentre of recorded earthquakes data. Quadrat analysis and the nearest neighbour analysis were done to find out the spatial pattern in the point distribution. In many other situations, it is also desirable to carry out such tests repeatedly as new point location data are collected, with the objective of detecting change as quickly as possible. For instance, it is of interest to detect changes in the spatial pattern of diseases (Farrington and Beale, 1998; Rogerson, 1997). Monitoring the residential locations of new customers is important for businesses to assess their markets and competition. Quick detection of changes in the pattern of criminal activity may lead to improved allocation of police resources (Rogerson et al., 2001).

In summary, studies on spatial and statistical modeling on urban systems exist in the literature. However, an integrated spatial and statistical analysis associated with water mains failure has not received much attention. In this chapter, we develop and evaluate a procedure based on spatial statistics to synthesize the geographical pattern of pipeline failure across the pilot area.

\subsection{Methodology}

The methodology followed in this research can be described in the following steps:

1. Collection of case study water mains failure information through a combination of different databases including a water distribution failure database and customized ArcView/GIS. 
2. Geo-code failures in a GIS database: Convert each failure to a point object based on its location as referenced by address (streets/intersection).

3. Carry out various spatial statistical operations like calculation of mean center, weighted mean center, standard distance, standard deviation ellipse.

4. Finally, perform quadrat and the nearest neighbour analysis on the failure dataset to find out the spatial point pattern.

\subsubsection{Data Collection}

One of the most critical and expensive parts of any pipeline failure program is the collection of reliable data on the failure distribution of the water system. Within the scope of this project, failures data in the central part of Sanandaj city have been recorded since 1995 (Figure 25.1). It contains both map data (depicting location of failures) and attribute data (describing physical characteristics of each failure). Traditional sources of spatial data including archival sources such as field notebooks, census material, maps and as-built drawings were computerised from paper archives five years ago. The data gap was further reduced based on interviews with Sanandaj Water and Wastewater Utility (SWWU) staff.

\section{Study area}

The area chosen for this study, Sanandaj city in the west of Iran (approximately longitude: 46.99 and latitude: 35.32), is an extraordinary case due to the unusually high number of main breaks (two failures/kilometre/year). It has a young distribution system, $70 \%$ of it built since 1960 and $30 \%$ since 1995 . The SWWU operates a water distribution system covering approximately $90 \mathrm{~km}^{2}$ and providing retail water service to more than 210,000 people. Average water use was in the range of 35,000$45,000 \mathrm{~m}^{3} /$ day. Two major sources of drinking water, surface water and groundwater, supply the network. Treated water is delivered from the water treatment plant to the ground reservoirs by pumping. Water storage in the distribution system is provided by twelve elevated storage facilities to maintain pressure levels in the gravity-pressure system. The pressure in the network is between 2 and 6.5 bar.

This work focuses on the pilot area which was found in a high-density residential and commercial area in the center of city. The pilot area serves 
approximately 11000 connections, corresponding to 554 mains segments. Its distribution system consists of nearly $56.7 \mathrm{~km}$ of water mains from 63 to $800 \mathrm{~mm}$ in diameter made variously of cast iron (13\%), ductile iron $(21 \%)$, asbestos cement $(36 \%)$, and polyethylene $(30 \%)$. In the past ten years, the pilot area has experienced approximately 35 to 45 failures per year. Polyethylene and cast iron pipes had a higher number of failure than other materials.

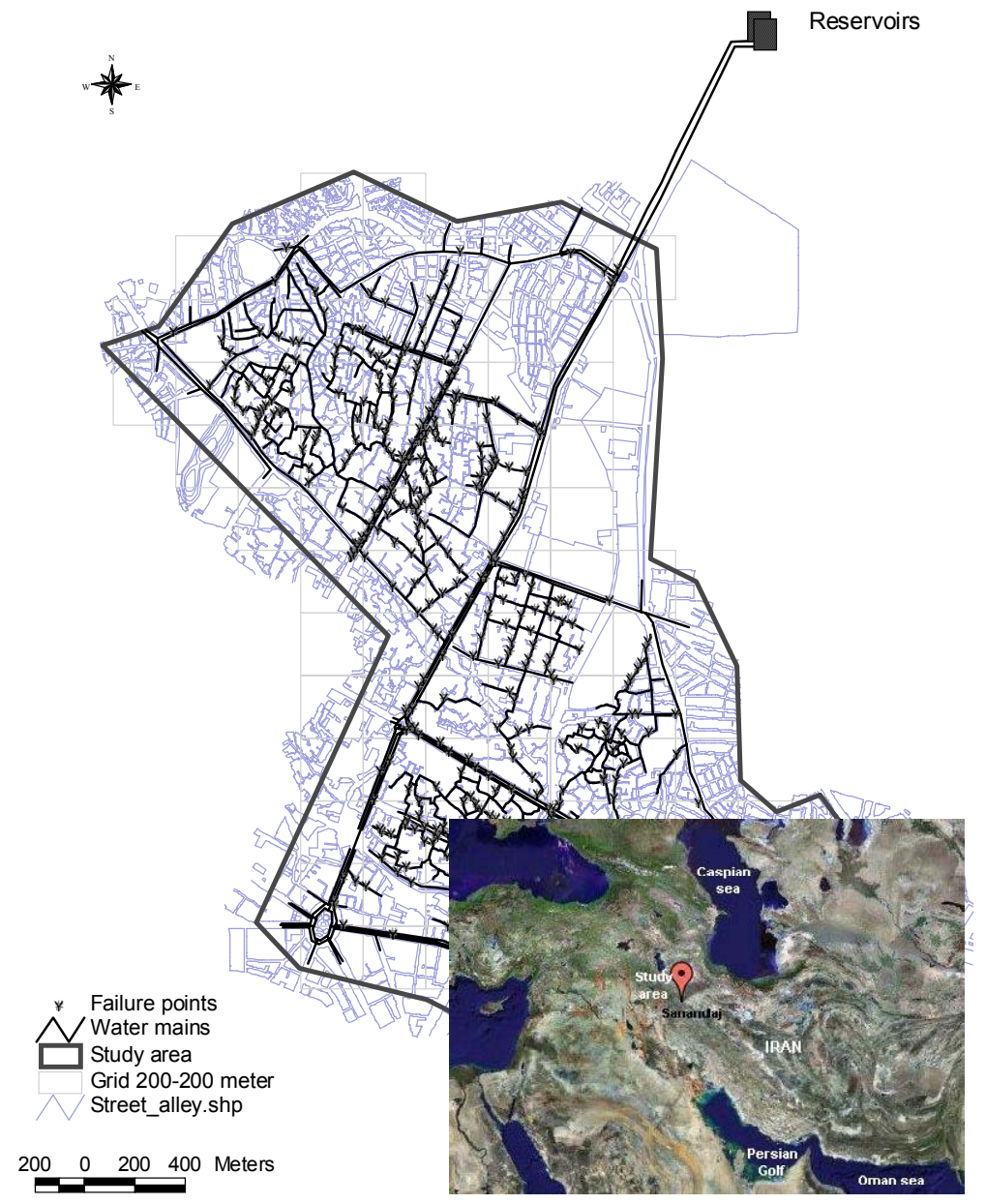

Figure 25.1 GIS layers and point location of mains failures in study area. 


\section{Water Mains Failure Database}

Data used in this chapter were collected for the period 1995-2004 by the Repair Division of the SWWU. User-friendly applications were programmed into Access $97^{\mathrm{TM}}$ to record data for each failure case. The database contains daily recorded information of contractors who repaired damaged water mains. In MS-Access ${ }^{\mathrm{TM}}$, the electronic forms provide ways of collecting and documenting main breaks and pipe condition information using pick lists and standard input. Therefore, it improves the paper based processes and reduces data entry errors, data redundancy and preserves data integrity (Heise and Takara, 2003).

The Access ${ }^{\mathrm{TM}}$ file has three basic tables, pre-constructed queries and macros, and customized menu items to store the data and report query results. The pipeline's table includes ID, material, diameter, wall thickness, depth of burial, category of upper street, age, segment length and hydraulic pressure. Failure attributes contain information concerning positional and temporal data such as $x / y$ coordinate, date of occurrence and repair, possible cause and type of failure. Finally, administrative data will include character of system operator and repairing crews, security and classifying codes, equipment and material used as well as cost evaluation. The three tables have been joined via the common $I D$.

\section{Importing data from the failure database into GIS}

The main graphical user interface (GUI) for collecting water mains failure data is a standard ArcView ${ }^{\mathrm{TM}}$ 3.2a software and Access database. This integration gives all statistical and customizing capabilities of Access and all the functionality of ArcView.

In terms of GIS, water main failure is a phenomenon which can be expressed through occurrences identified as points in space. Generally, failure location was reported by a crew through measuring the distance from the reference point using a tape measure. The crew would estimate the distance of the failure location from a known reference, normally the nearest street, alley or intersection. Clearly the estimation was subject to errors but this work tried to minimize it. Technological advancement calls for a need to revolutionize this approach and there is a strong recommendation that global positioning system (GPS) receivers be adopted for capturing failure location in SWWU. Failure mapping starts with geocoding process that matches an address of breakage to a physical location (as a dot) along a street. Each failure point is converted manually into coordinate locations via street or mailing address matching. At a scale of 1: 2000, the reference map provides 
a suitable level of detail to approximately locate property boundaries, streets and distribution mains features. Accordingly, the water mains network and failures were digitized visually against a backdrop of streets, property parcel and buildings. The process of identifying the coordinates of a failure point in ArcView 3.2a, is to use "getx" command for the x-coordinate and "gety" command for the y-coordinate. For means of the coordinate projection system, this chapter considered the UTM system. Both $x$ and $y$ are defined by distances in meters from an arbitrary reference point. These projected coordinates are added to the original data record and read directly into the spatial statistical program such as CrimeStat (Figure 25.2).

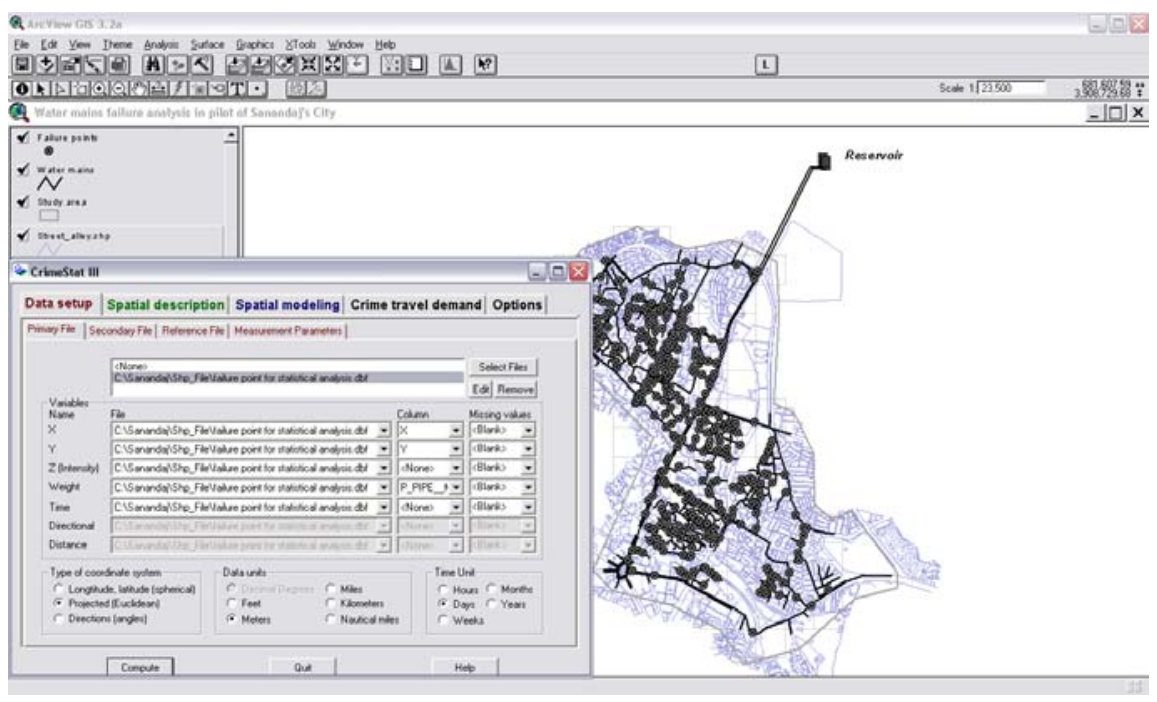

Figure 25.2 The interface of CrimeStat package and GIS.

When an address list is transformed into a set of coordinate points, corresponding failure attributed data are then imported from Access into ArcView. The platform in ArcView is connected with external databases using the SQL connection feature. In order to combine and join data to a point shapefile of locations according to each failure, a common field such as failure ID should exist in both tables. By joining this field between tables, data can be retrieved from each table and combined into another table. The data is then saved as a shapefile in GIS. In fact, each failure on the pipeline 
can be created by a common $I D$ number in ArcView and Access databases. Therefore, the related non-spatial data about failure is stored in MS Access and graphical information is established in ArcView. Each record in the tabular database is then connected with a failure point in GIS. Location of all of reported breaks for the 10-y data period were plotted on the map by small black dots (Figure 25.1). At first glance, this map indicated visually that break occurrence is not uniformly distributed across the service area but that certain areas have higher densities than others.

To provide a set of spatial statistics, the CrimeStat III program and ArcView 3.2 were applied. Most of the methods in CrimeStat are applicable to the analysis of spatial failure data. This program analyzes point data, defined geographically by $x$ and $y$ coordinates (Levine, 2004). These $x / y$ coordinates represent a single location where an incident occurred on water mains. By means of the graphic user interface, as shown in Figure 25.2, the coordinates will be transferred to CrimeStat. The package read directly nonspatial data from "failure point.shp" layer and supports graphical outputs to ArcView.

\subsubsection{Analysis}

The analysis strategy was established by expression of failures as a point in space (Figure 25.3). Spatial analysis involves the analysis of data representing geographical features which have a locational attribute such as absolute location (coordinates) or relative positioning (distance). Prior to point pattern analysis, a set of descriptive spatial statistics e.g. spatial measures of central tendency and dispersion were done. Additionally, spatial analysis applied to point data typically involves the analysis of point distributions and the relationship between point distributions and other spatial features. The objective of the analysis would be to determine if the point pattern is "regular", "random", or "clustered". Two basic techniques are used based on (i) counting of cases within small squares (quadrat analysis) and (ii) measuring distance to the nearest case (nearest-neighbor analysis).

All spatial statistical analysis was performed using the CrimeStat III software package. It is a freely available package that provides a variety of tools for the spatial analysis of crime incidents or other types of applications involving point locations, such as the location of pipeline failures. 


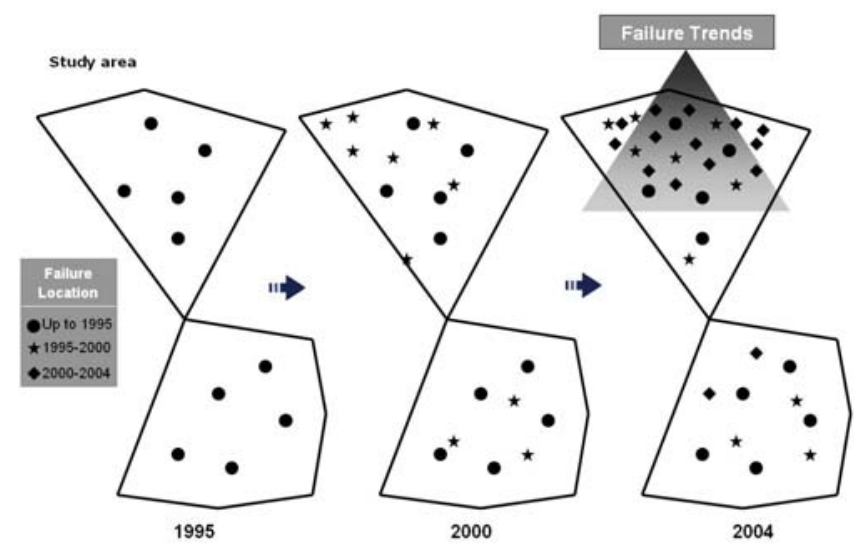

Figure 25.3 Temporal and spatial trends in water mains failures.

\section{Central Tendency Scores}

Since points were used to indicate the spatial occurrence of pipeline failure, to get an idea about the overall pattern of failure distribution in the study area, some basic descriptive statistics were generated. The measures of spatial central tendency, or centrographic measures, like mean and weighted mean center scores were determined for analysis of the failure distribution.

The most basic form of statistical analysis for measurement of central tendency in geographical data is called Mean Center or balance point. It is concerned with the center of a geographic point dataset in which the points in the distribution represent occurrences of pipeline failure in different periods of time. By taking the $x$ and $y$ coordinates of each point, the bivariate mean center is determined using the expression:

$$
\left(\bar{x}_{m c}, \bar{y}_{m c}\right)=\left(\frac{\sum_{i=1}^{n} x_{i}}{n}, \frac{\sum_{i=1}^{n} y_{i}}{n}\right)
$$

where:

$$
\begin{aligned}
\bar{x}_{m c}, \overline{\mathrm{y}}_{\mathrm{mc}} & =\text { mean center coordinates } \\
x_{i}, y_{i} & =\text { coordinates of each water mains failure location, and } \\
n & =\text { total number of failures. }
\end{aligned}
$$


The mean center computed from this formula, appears right in the middle of the geographic area (Figure 25.4).

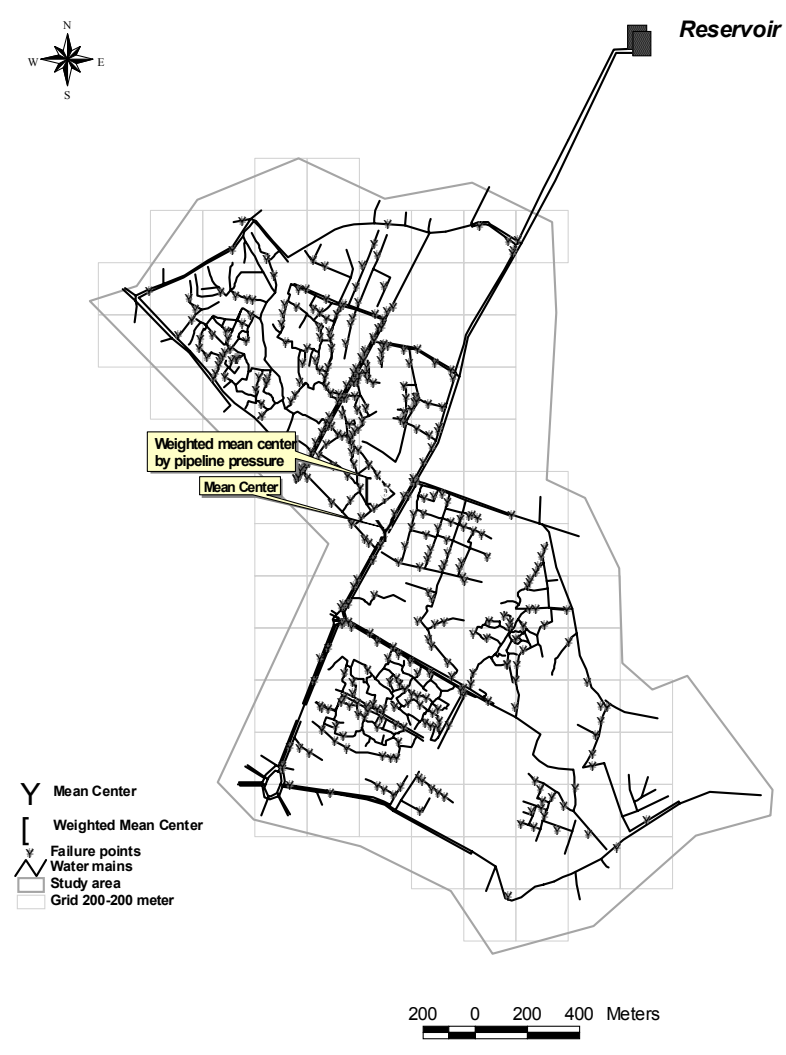

Figure 25.4 The scatter plot of failure points, mean center and weighted mean center by the pipeline pressure.

Moreover, the weighted mean center is calculated by weighting each coordinate by another variable, as below:

$$
\left(\bar{x}_{w m c}, \bar{y}_{w m c}\right)=\left(\frac{\sum_{i=1}^{n} w_{i} x_{i}}{w}, \frac{\sum_{i=1}^{n} w_{i} y_{i}}{w}\right)
$$


where:

$$
\begin{aligned}
\bar{x}_{w m c}, \overline{\mathrm{y}}_{\mathrm{wmc}} & =\text { weighted mean center coordinates }, \\
w_{i} & =\text { weight }, \text { and } \\
w & =\text { total weight. }
\end{aligned}
$$

Descriptive statistic for failure data showed that high pressure in the pipeline is the common cause of failure in the case study (Asnaashari et al., 2005). By multiplying pressure in the coordinates of each failure point, the weighted mean center would be mapped. Thus, water mains failure points affected by high pressure pull the weighted mean towards them. Figure 25.4 shows that the computed weighted center is to the northwest of the mean center. It might show that there is more pressure in the northwest portion of the study area. According to the topography and general slope of the city, and to local expert opinion, this is correct.

\section{Dispersion Scores}

Dispersion scores provide a unit measure of spread or variability of a failure distribution. This analysis addresses questions such as:

1. Do the failures cluster about their central point or do they spread out around it?

2. Where are the high or low concentrations of failure?

3. Is there a pattern to the failure data?

In spatial statistical analysis, standard deviation is expressed as standard distance. While standard deviation indicates how observations deviate from the mean, standard distance indicates how points in a distribution deviate from the mean center. Standard deviation is expressed in the units of observation values, but standard distance is expressed in distance units. In terms of its application, standard distance is usually used as the radius to draw a circle around the mean center to give the spatial spread of the point distribution it is based on (Saraf et al., 2003). The radius equal to $S D_{x, y}$ :

$$
S D_{x, y}=\sqrt{\frac{\sum_{i=1}^{n}\left(x_{i}-\bar{x}_{m c}\right)^{2}}{n-1}+\frac{\sum_{i=1}^{n}\left(y_{i}-\bar{y}_{m c}\right)^{2}}{n-1}}
$$

where:

$$
\begin{aligned}
x_{i}, y_{i} & =\text { coordinates of each water mains failure location, } \\
\bar{x}_{m c}, \bar{y}_{m c} & =\text { coordinates of mean center, and } \\
n & =\text { total number of failures }
\end{aligned}
$$


The computed radius is equal to $720.28 \mathrm{~m}$ (Figure 25.5). Essentially the average distance of points from the center provides a single unit measure of the spread or dispersion of water mains failure distribution.

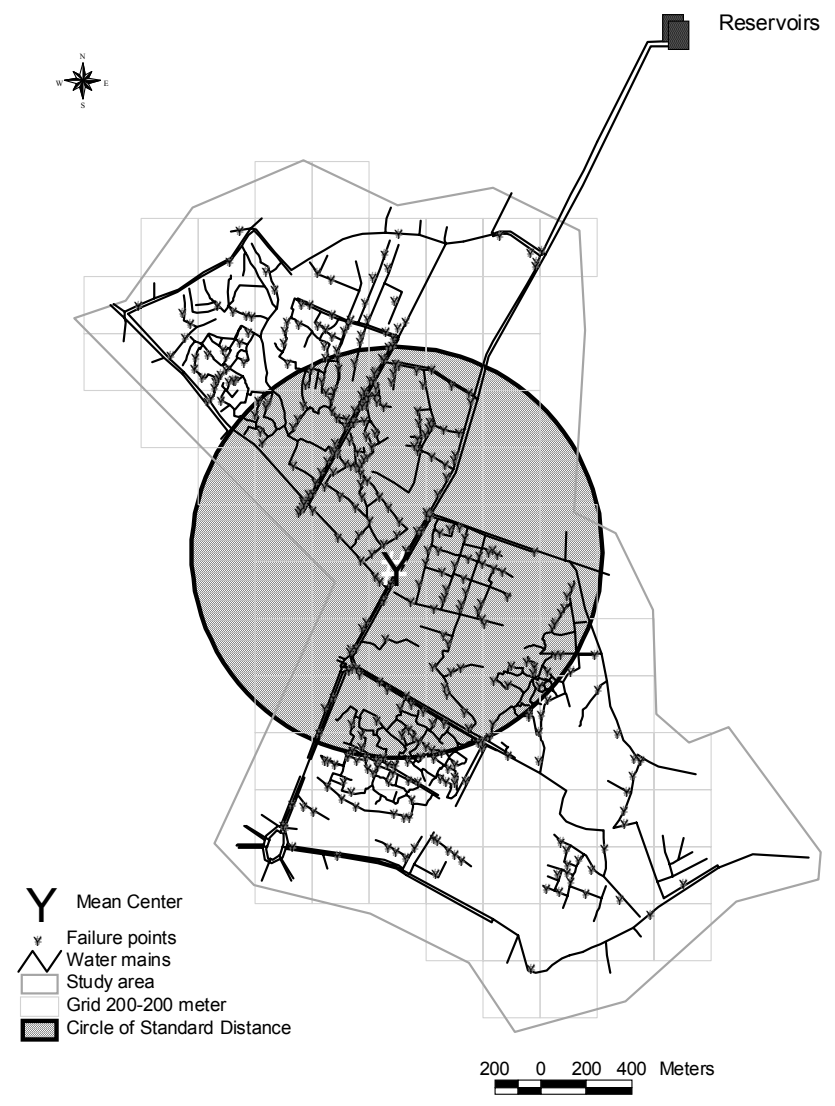

Figure 25.5 The circle represents the Standard Distance Deviation $\left(S D_{x y}=\right.$ Circle radius $\left.=720.28 \mathrm{~m}\right)$

While the standard distance deviation (SDD) is a good measure of the dispersion of the incidents around the mean center, it does not show the potential skewed nature of the data (anisotropy). The standard deviation ellipse gives dispersion in two dimensions and surrounds most of points. It can be a tool to explore variables that are affecting failure patterns. 
As seen in Figure 25.6, Standard Deviational Ellipse (SDE) is defined by three parameters: angle of rotation $(\theta)$, dispersion along major axis $\left(\delta_{x}\right)$, and dispersion along minor axis $\left(\delta_{y}\right)$. The major axis defines the direction of maximum spread of the distribution and minor axis is perpendicular to it and defines the minimum spread.

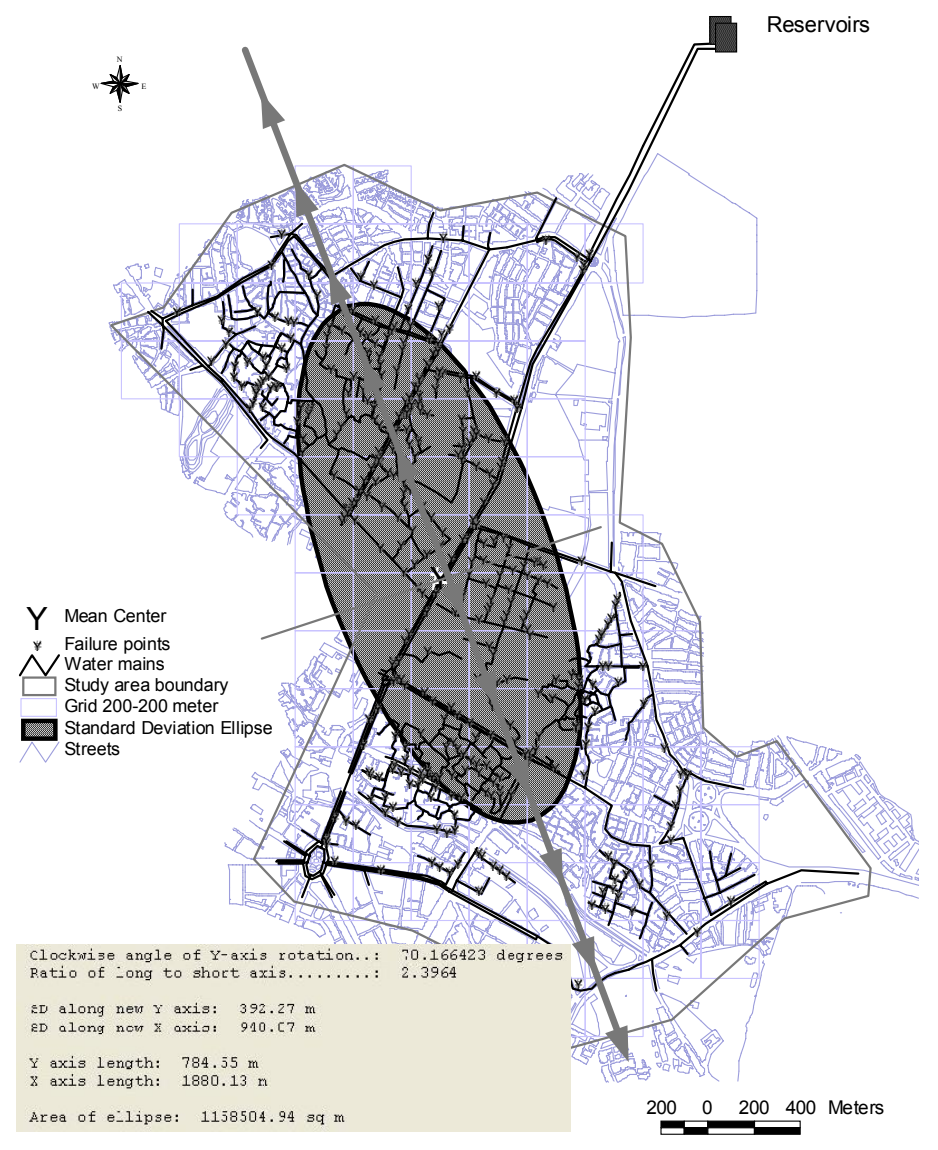

Figure 25.6 Standard Deviational Ellipse around the mean center of failure locations. 
The angle of rotation, $\theta$, which has the definition below:

$$
\begin{aligned}
& \tan \theta=\frac{\left(\sum_{i=1}^{n} x_{i}^{\prime 2}-\sum_{i=1}^{n} y_{i}^{\prime 2}\right)+\sqrt{\left(\sum_{i=1}^{n} x_{i}^{\prime 2}-\sum_{i=1}^{n} y_{i}^{\prime 2}\right)^{2}+4\left(\sum_{i=1}^{n} x_{i}^{\prime} \sum_{i=1}^{n} y_{i}^{\prime}\right)^{2}}}{2 \sum_{i=1}^{n} x_{i}^{\prime} \sum_{i=1}^{n} y_{i}^{\prime}} \\
& x_{i}^{\prime}=x_{i}-\bar{x}_{m c} \quad, \quad y_{i}^{\prime}=y_{i}-\bar{y}_{m c}
\end{aligned}
$$

The deviations along the $x$ and $y$ axes are defined as:

$$
\delta_{x}=\sqrt{\frac{\sum_{i=1}^{n}\left(x_{i}^{\prime} \cos \theta-y_{i}^{\prime} \sin \theta\right)^{2}}{n}}, \quad \delta_{y}=\sqrt{\frac{\sum_{i=1}^{n}\left(x_{i}^{\prime} \sin \theta-y_{i}^{\prime} \cos \theta\right)^{2}}{n}}
$$

\section{Point Pattern Analysis}

Point pattern analysis is concerned with attempting to determine whether the distribution of points is random or whether it either clusters or is evenly distributed. This work was carried out two ways to assess the distribution of water mains failure : nearest neighbor analysis and quadratic analysis.

\section{Nearest Neighbor Analysis}

The nearest neighbor index $(R)$ provides an approximation about whether points are more clustered or dispersed than would be expected on the basis of chance (Diggle, 1983). It compares the average distance of the nearest other point (nearest neighbor) with a spatially random expected distance. The expected distance is given by:

$$
\overline{\mathrm{NND}}_{R}=\frac{1}{2 \sqrt{\text { Density }}} \quad \text { Density }=\frac{n}{A}
$$


Thus:

$$
R=\frac{\overline{N N D}}{\overline{N N D}_{R}} \quad, \quad \overline{N N D}=\frac{\sum_{i=1}^{n} N N D}{n}
$$

where:

$$
\begin{aligned}
n & =\text { number of failure points in the distribution, } \\
A & =\text { the size of study area, } \\
R= & \text { the ratio of the observed to the expected distance, } \\
N N D= & \text { distance between each point and its nearest } \\
& \text { neighbor, } \\
\overline{N N D}= & \text { the observed mean distance between nearest } \\
& \text { neighbor. } \\
\overline{N N D}_{R}= & \text { the expected value of the nearest neigbor distance in } \\
& \text { a random pattern }
\end{aligned}
$$

For these data, the mean nearest neighbor distance and expected mean nearest neighbor distance are calculated 36.2 and 55.9, respectively. Since the analyzed mean distance is shorter than the mean distance for the random pattern, the pattern is clustered. In fact, for this purpose, the $R$ ratio is used. $R$ values $<1$ indicate clustering, since the observed mean distance between neighboring points is less than that expected in a random pattern. The minimum value of $R$ is zero, which occurs when all points are at a single location. The theoretical maximum of $R$ is equal to 2.149, which occurs when points are maximally dispersed. Since the nearest neighbor index $R$ is equal to 0.655 , the distribution of pipeline failure is more clustered than random.

To help place confidence in the nearest neighbor index result, a test statistic can be calculated as follows (Cressie, 1993):

$$
Z_{n}=\frac{\overline{N N D}-\overline{N N D_{R}}}{\sigma_{\overline{N N D}}}
$$

where the standard deviation of mean distance between nearest neighbors in a random pattern is: 


$$
\sigma_{\overline{N N D}}=\frac{0.26136}{\sqrt{n \times(\text { Density })}}
$$

If values of the $Z_{n}$ statistic are in the interval from -1.96 to +1.96 , the pattern is random (at a confidence level of $95 \%$ ). If the $Z_{n}$ value exceeds +1.96 , the pattern is regular. If the $Z_{n}$ value is lower than -1.96 , the pattern is clustered (Rogerson et al., 2001). The value of $Z_{n}=-13.1$ was arrived at by using Equation 25.8 which indicates a clustered pattern exists.

\section{Quadratic Analysis}

Quadrat analysis is one of two most commonly used tools for analyzing the dispersion of points. At first, the study area was sub-divided into regular grid squares and the number of occurrences of water mains failure in each square is counted. The formula for determining the optimal quadrat size (length of the square side) is:

$$
\text { quadrat size }=\sqrt{\frac{2 \times A}{n}}
$$

where $\mathrm{A}$ is the size of the study area and $\mathrm{n}$ is the number of points.

The optimal quadrat size was determined here to be $200 \mathrm{~m}$. Figure 25.7 depicts the classification of quadrat according to the number of points in each cell. Then, the variance/mean ratio $(V M R)$ was used to compare the obtained empirical frequency with the theoretical frequency for the random pattern obtained from the Poisson distribution. In a Poisson distribution, the mean and variance are equal.

According to following expression, if the distribution is random the $V M R$ is about 1.0. Larger values $(V M R>1.0)$ correspond to existence of "clumps" (spatial clusters). Smaller values $(V M R<1.0)$ correspond to a moreuniform-than-random distribution:

$$
V M R=\frac{V A R}{M E A N}
$$




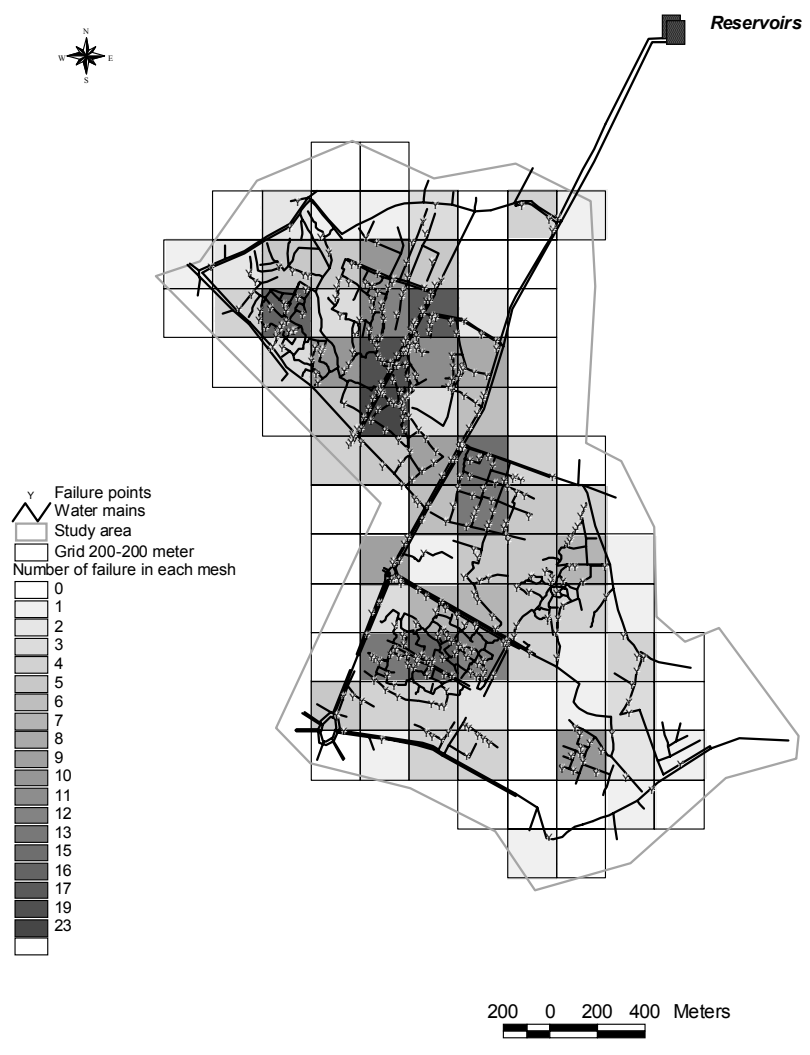

Figure 25.7 Number of water mains failure point in each cell.

Thus :

$$
\mathrm{VAR}=\frac{\sum_{i=1}^{n}\left(x_{i}-\bar{x}\right)^{2}}{n-1} \quad, \quad \operatorname{MEAN}=\overline{\mathrm{x}}=\frac{\sum_{i=1}^{n} x_{i}}{n}
$$

where:

$x_{i}=$ number of water mains failure points in cell $i$, and $n=$ total number of cells. 
We obtain: $V M R=5.9$; since $V M R$ is greater than 1, the point pattern can be considered as more clustered than random. Rather than base a conclusion on variance/mean ratio, we can compare observed frequencies in the quadrats with random frequencies realized from a homogenous Poisson process. Therefore, chi - statistic $\left(\chi^{2}\right)$ test was calculated as follow:

$$
\chi^{2}=\sum \frac{\left(x_{i}-\bar{x}\right)^{2}}{\bar{x}}
$$

where:

$$
\begin{aligned}
x_{i} & =\text { number of points in each quadrat, and } \\
\bar{x} & =\text { mean number of points per quadrats. }
\end{aligned}
$$

The significantly large $\chi^{2}$ value, 562.5 , indicates that the distribution is not uniform and that there may be some underlying process causing the nonuniformity (clustering).

\subsection{Results and Discussions}

This work summarized the point distribution of water mains failures through a set of descriptive spatial statistics. These statistics describe the degree of spatial variability of failures across study area. By incorporating the $\mathrm{X}$ and $\mathrm{Y}$ values for locations, we generated two most common quantities, mean and weighted mean center, for measuring the central tendency of failures. The chapter primarily plotted the geographical center on the map which appears right in the middle of the geographic area. In a weighted mean computation, we weight the $\mathrm{X}$ and $\mathrm{Y}$ coordinates by the failure attributes. Since hydraulic water pressures vary in different locations of study area, water mains in high pressure region (in excess of 3.5 bar) provide a greater probability for failure. This pressure comes from the calibrated hydraulic model and was stored in the failure data base. By multiplying pressure of water mains with each coordinate of related failure $(\mathrm{x}, \mathrm{y})$, the weighted mean center was computed. Thus, water mains failure points which were affected by high pressure pull the weighted mean toward them. But the results did not show much variance in its location, compared to the unweighted mean center (Figure 25.4). Therefore, it would indicate that the entire area is continuously being struck by high pressure in the water mains network. 
Otherwise, the weighted mean center could have been at significant distance apart from the unweighted mean center. Further, this research evaluated dispersion scores of the data set. The circle of standard distance deviation shows that water mains failures are located at the middle part of the study area. The greater the standard deviation distance, the more dispersed the failure incidents. Next, the standard deviation ellipse for the data of failure is drawn with the calculated major and minor axes that show the directions of maximum and minimum spread of locations, respectively. It is notable that the orientation of the major axis of the standard deviation ellipse is more parallel with the trend of the major traffic lines in the pilot area. Depicted in Figure 25.6 is simply the distribution of the failure points and standard deviation ellipse.

In this chapter, we have calculated a point pattern statistic through nearest neighbor and quadrat methods. The pattern analysis began with an exploration of nearest neighbour distances. The Nearest Neighbor Index (NNI) is a second order distance statistic that was measured based on the minimum inter-feature distance and compares this to what would be expected on the basis of chance. The mean nearest neighbor distance and expected mean nearest neighbor distance were determined as 36.2 and $55.9 \mathrm{~m}$, respectively. Then, the NNI results for this analysis $(N N I<1.0)$ and value of $Z_{n}$ - test $\left(Z_{n}=-13.1\right)$ showed that many of failure points are concentrated close together (clustered). For further analysis, we then applied quadrat method for analyzing the dispersion of failure points. The results of variance mean ratio (VMR) and goodness-of-fit statistics ( $\chi^{2}$ analysis), also showed that the distribution of water mains failure is more clustered than random. Clearly, evidence of spatial clustering in such a point pattern could be result of environmental, operational, or physical factors.

\subsection{Conclusions}

This chapter details a framework for better understanding the spatial characteristics of water mains failure based upon the use of geographical information systems and quantitative techniques in the City of Sanandaj. Simple spatial statistics and point pattern analysis were carried out on the location of failure points. The results of quadrat analysis and nearest neighbour analysis display the water mains failures tend to occur in clusters. Water utilities define the cluster areas as a subregion of the city with a greater probability for another failure and also an area suitable for failure 
surveillance and leakage detection. Additionally, identification of clusters of a certain failure in a specific location is useful in determining if the failure is caused by some phenomenon at that location, although the approach described here focuses upon changes in geographical patterns only. Thus it does not signal either an increase or decrease in the rate of failure that may have taken place. Areas with greater pipe length per unit area, for example, may have more breaks. This should not be viewed as a weakness of the method; the spatial pattern detection method is designed to do exactly what its name implies, and should of course be combined with other appropriate analytical tools as well as expert knowledge that achieve other objectives.

Finally, the use of this method has also a lot of benefit in the ranking of rehabilitation projects. Consequently, companies routinely target their mains renewal projects in these areas.

In summary, application of the approach has been successfully used in analysis of mains bursts in water reticulation systems. However, further research is recommended in several cities and real data to evaluate the results, with the objective of identifying some common trends and geographical correlation between pipe failure and spatial characteristics.

\section{Acknowledgments}

The authors would like to thank Dr. Motiee H., Soltani S., Pakrouh S., and the Sanandaj Water and Wastewater Utility (SWWU) for providing the pipelines failure data used in this study. Funding was provided by Power and Water University of Technology in Tehran, IRAN (PWUT) and Laboratory of Mechanic in Lille, FRANCE (LML).

\section{References}

Asnaashari, A., Shahrour, I., Haji Sotudeh, M., and Soltani, S. (2005). Statistical investigation of failure of water distribution system: Application on the city of SANANDAJ in IRAN. Conference on Urban Engineering, Lille, France.

Clark, P. J., and Evans, F. C. (1954). Distance to nearest neighbor as a measure of spatial relationships in populations. Ecology 35: 445-453.

Cliff, A. D. and Ord, J. K. (1981). Spatial Processes: Models and Applications. Pion, London.

Cressie, N. A. C. (1993). Statistics for Spatial Data,Wiley, New York. 
Diggle, P. J. (1983). The statistical analysis of spatial point patterns. London: Academic Press.

Farrington, C. P., \& Beale, A. D. (1998). The detection of outbreaks of infections disease. In L. Gierl, A. D. Cliff, A. Valleron, P. Farrington, \& M. Bull, GEOMED 97, international workshop on geomedical systems (pp. 97-117). Stuttgart: B. G. Teubner.

Harvey, D.W. (1966) Geographical processes and point patterns: testing model of diffusion by quadrat sampling. Transactions of the Institute of British Geographers 40: 81-95.

Heise, S., and Takara, H. (2003). How to streamline water main failure and condition assessment using geospatial technology. Proc., Geospatial Information \& Technology Association (GITA).

Hu, Y., and Hubble, D. (2005). Failure conditions of asbestos cement water mains in regina, Canadian Society of Civil Engineering (CSCE) 33rd Annual Conference, Toronto, Ontario, Canada, June 2-4, 2005.

Levine, N.,. Kim, K.E., and Lawrence, H. N. (1995). Spatial analysis of Honolulu motor vehicle crashes: I. Spatial patterns. Accident Analysis \& Prevention, 27 (5), 663-674.

Levine, N. (2004). CrimeStat III: A Spatial Statistics Program for the Analysis of Crime Incident Locations - version 3.0 : User's Manual; Ned Levine \& Associates: Houston, TX/ National Institute of Justice: Washington, DC.

Lienyao, L., Chungsying L., and Shyang-Lai K. (2004). Spatial Diversity of Chlorine Residual in a Drinking Water Distribution System. Journal of Environmental Engineering. Volume 130, Issue 11, pp. 1263-1268.

Goulter, I., Davidson, J., \& Jacobs, P. (1993). Predicting water-main breakage rates. Journal of Water Resources Planning and Management, 119(4), pp. 419-436.

Mantel, M. (1967). The detection of disease clustering and a generalised regression approach. Cancer Research 27, pp. 209-220.

O'Day, D. K., Weiss, R., Chiavari, S., \& Blair, D. (1989). Water main evaluation for rehabilitation/replacement. Guidance manual of AWWA Research Foundation, 182.

Saraf, A. K., Sarma, B., and Chandramani (2002). Spatial statistical technique in relating earthquake epicentres with structural features. Retrieved June, 2002, from http://www.gisdevelopment.net/application/natural_hazards/earthquakes/nheq0010.htm

Rogerson, P. (1997). Surveillance systems for monitoring the development of spatial patterns. Statistics in Medicine, 16, 2081-2093.

Rogerson, P., \& Sun, Y. (2001). Spatial monitoring of geographic patterns: an application to crime analysis. Computers, Environment and Urban Systems, Vol. 25, pp. 539-556. 
